\title{
Lagrangian descriptors and the assessment of the predictive capacity of oceanic data sets
}

\author{
C. Mendoza ${ }^{1}$, A. M. Mancho ${ }^{2}$, and S. Wiggins ${ }^{3}$ \\ ${ }^{1}$ ETSI Navales, Universidad Politécnica de Madrid, Av. Arco de la Victoria 4, 28040 Madrid, Spain \\ ${ }^{2}$ Instituto de Ciencias Matemáticas, CSIC-UAM-UC3M-UCM, C/ Nicolás Cabrera 15, Campus Cantoblanco UAM, \\ 28049 Madrid, Spain \\ ${ }^{3}$ School of Mathematics, University of Bristol, Bristol BS8 1TW, UK
}

Correspondence to: A. M. Mancho (a.m.mancho@icmat.es)

Received: 17 December 2013 - Revised: 29 April 2014 - Accepted: 6 May 2014 - Published: 18 June 2014

\begin{abstract}
We use a recently developed Lagrangian transport tool, Lagrangian descriptors, to compare the transport properties of data distributed by AVISO and numerical simulations obtained from the HYCOM model in the Yucatán-Florida current system. Our data correspond to the months from June through August 2010. Structures obtained from HYCOM are noisier than those from AVISO; however, both AVISO and HYCOM succeed in identifying Lagrangian structures that influence the paths of drifters, such as eddies, currents, lobes, etc. We find evidence in which AVISO gives the positions of important hyperbolic trajectories in a manner that is inconsistent with the trajectories of the drifters, while for the same examples HYCOM succeeds to this end.
\end{abstract}

\section{Introduction}

This research is concerned with the use of theoretical and computational techniques coming from dynamical systems theory that are used to assess the performance of gridded velocity fields originating from realistic ocean models and data sets. The development of the Lagrangian aspects of fluid transport in the context of oceanic flows has received a great deal of motivation from the popularity and from the successes of the dynamical systems approach to fluid transport in the 1980s and 1990s. Background and reviews can be found in Jones and Winkler (2002), Mancho et al. (2006b), Wiggins (2005), and Samelson and Wiggins (2006). Hyperbolic trajectories and their stable and unstable manifolds provide a generalization to the time-dependent setting of saddletype stagnation points and their separatrices. In general, in- variant manifolds are material curves (or surfaces in three dimensions; see for instance Bettencort et al., 2012; Froyland et al., 2012) that form a Lagrangian skeleton that governs the spatiotemporal evolution of the transport of fluid particles. Methods for computing hyperbolic trajectories and their stable and unstable manifolds for velocity fields defined as finite time data sets can be found in Miller et al. (1997), Malhotra and Wiggins (1998), Haller (2000), Mancho et al. (2003), Mancho et al. (2004), and Branicki and Wiggins (2009). Finite time Lyapunov exponents (FTLEs) and finite size Lyapunov exponents (FSLEs) have proved useful for determining proxies for invariant manifolds (see, e.g., Pierrehumbert, 1991; Haller and Yuan, 2000; Haller, 2001a; Haller, 2001b; Shadden et al., 2005; Lekien et al., 2007; and d'Ovidio et al., 2004). In this paper we use a recently developed Lagrangian tool, referred to as a Lagrangian descriptor (Mancho et al., 2013), that is described in detail in Sect. 5.

Nowadays, a huge amount of "realistic" ocean data supplied by direct observations is available. Surface currents are measured either through high-frequency coastal radars and satellite altimeters or, alternatively, through measurements with in situ devices (i.e., acoustic Doppler current profilers, ADCPs, mounted on vessels, fixed moorings, gliders, drifters). Many of these data are assimilated into numerical oceanic models, which in turn provide outputs of velocity and other scalar fields in full 3-D domains. Obtaining accurate representations of oceanic currents is of tremendous importance not only for a better management of catastrophic events, such as, for instance, contaminant dispersion, but also for their direct impact on marine life and fishing banks, for 
optimizing shipping routes, for search and rescue operations, etc.

Lagrangian techniques have been applied to different oceanic data sets for purposes related to the goals of this work. For instance, the correlation between Lagrangian structures obtained from radar data in different ocean areas is discussed by Shadden et al. (2009) and Haza et al. (2010). Kuznetsov et al. (2002) have examined the role played by Lagrangian structures in the Gulf of Mexico. In particular, they have analyzed data from the Colorado University Princeton Ocean Model and have compared the geometry of the dynamics with simultaneous trajectories of drifters measured in an independent way. Indirectly the agreement between the drifter evolution and the Lagrangian patterns serves as a confirmation of the model quality. The ability of coastal models to capture the true Lagrangian structure of ocean flows has been discussed also in Haza et al. (2007) by using FSLEs, and they concluded that this model-derived Lagrangian metric was capable of predicting the general fate of observed drifter pairs. Griffa et al. (2013) point out that accuracy of Lagrangian diagnosis is directly tied to the accuracy of the Eulerian velocity and perform experiments that prove that the Lagrangian diagnosis relying on Eulerian fields from accurate operational coastal models can successfully guide real-time drifter launch strategies. In turn hyperbolic trajectories detected by Lagrangian techniques have guided drifter launch strategies for a better Lagrangian assimilation (Molcard et al., 2006).

Lagrangian techniques have been used to provide feedback that improve numerical models. For instance Titaud et al. (2011) implement an assimilation method that uses correspondence between advected passive scalars and FTLEs and vectors. Nencioli et al. (2011) propose an iterative method that uses satellite data, ADCP and drifters to improve the detection of Lagrangian coherent structures (LCSs). Nencioli et al. (2013) use FTLE to estimate horizontal eddy diffusivity, a parameter required by numerical simulations.

Lagrangian techniques have also been used in the past to compare predictions based on different data sets. Beron-Vera et al. (2010) evaluate the surface ocean mixing predicted by two different altimetry AVISO products, REF and UPD, constructed with, respectively, two and four satellite altimeters. Their analysis is based on a mixing characterization obtained from statistics based on FTLE. They also compare the quality of the data based on the agreement between a drifter trajectory and the Lagrangian skeleton supplied by the FTLE. With their analysis they improve the previous purely Eulerian analysis of the data (Pascual et al., 2006); however, a weakness of their approach is that FTLEs develop spurious structures that have no Lagrangian interpretation (Branicki and Wiggins, 2010; Mancho et al., 2013), and this could have biased their mixing diagnosis.

In this article we analyze the correlation between drifter trajectories and Lagrangian structures in the Gulf of Mexico obtained from two data sets: one comes from a numer- ical simulation and the second from satellite altimetry. The ocean model that we have considered is the HYCOM model (Bleck, 2002; Chassignet et al., 2007); as for altimeter data, we consider the AVISO product UPD, which is reconstructed from four satellites. In order to perform our analysis, we use a recently developed Lagrangian tool (Mendoza and Mancho, 2010; Mendoza et al., 2010; Mancho et al., 2013), the socalled $M$ function or Lagrangian descriptor. We expect that the sharp outputs obtained from this tool as confirmed in de la Cámara et al. (2012) and Mancho et al. (2013) will help us to obtain accurate conclusions from our analysis. The agreement between the drifters and the obtained geometry serves as a benchmark for the predictive capacity of the data sets.

This paper is organized as follows. Section 2 describes the data sets used to perform the analysis, which includes the details of the drifters that we have considered. Section 3 provides a description of the ocean area under study. Section 4 describes the construction of a dynamical system from the data set. Section 5 describes the Lagrangian descriptors used to reveal the geometry of the dynamics. Section 6 describes the results, and finally Sect. 7 discusses the conclusions.

\section{Data sets}

In this section we give a brief description of the types of data used to study the flow in the particular regions that we consider.

\subsection{HYCOM}

The Hybrid Coordinate Ocean Model (HYCOM) (Bleck, 2002; Chassignet et al., 2007) is part of the US Global Ocean Data Assimilation Experiment (GODAE) within the National Ocean Partnership Program (NOPP). This model uses the Navy Coupled Ocean Data Assimilation (NCODA) system: satellite sea-surface height and temperature data are assimilated, and the model is forced by surface winds and airsea fluxes. Specific details can be found at http://hycom.org/ dataserver/goml0pt04. The output of this model has been previously used to study transport processes in the Gulf of Mexico by Olascoaga (2010), Mezic et al. (2010), and Sulman et al. (2013).

In particular, we have used experiment 31.0 (http://hycom. org/dataserver/goml0pt04/expt-31pt0), which provides data from 2009 to the present. The experiment has $1 / 25$ degree equatorial resolution and latitudinal resolution of $1 / 25^{\circ} \mathrm{cos}$ (lat). This represents approximately a grid size at midlatitudes of $3.5 \mathrm{~km}$. The experiment uses 20 layers along the vertical coordinate and is carried out in a domain in which latitudes range from 18.09 to $31.96^{\circ}$ and the longitude ranges from 98.0 to $76.4^{\circ} \mathrm{W}$. The data are provided hourly.

Our calculations are performed in the fourth layer, which corresponds to a depth of $30 \mathrm{~m}$. This is an appropriate choice, as it mitigates excess noise of the data in the surface layer. 
A similar depth choice for this model is used, for instance, in Sulman et al. (2013) or for CUPOM (Colorado University Princeton Ocean Model) in Branicki and Kirwan (2010). In the latter work the authors show how the resulting Lagrangian structure is close to 2-D surfaces that extend nearly vertically into the water column, thus confirming that the choice of a deeper layer just affects the noise on the observed structures. We also note that continent shapes at $30 \mathrm{~m}$ depth are not the same as those at $0 \mathrm{~m}$ depth, for which we have more experience.

\subsection{AVISO}

Surface velocity fields are obtained from satellite altimetry and produced by Ssalto/Duacs and distributed by AVISO, with support from Cnes (http://www.aviso.oceanobs.com/ duacs/).

The data are given on a spatial grid of $1080 \times 915$ points (longitude/latitude). The latitude ranges from $82^{\circ} \mathrm{S}$ to $81.9746^{\circ} \mathrm{N}$ using a Mercator projection, and the longitude ranges from 0 to $359.667^{\circ}$ using a uniform grid. The spatial precision is thus $1 / 3^{\circ}$ at the Equator and is provided with daily frequency.

AVISO data are nowadays a standard source for analysis of transport processes in the ocean, not only in the area of interest in our study in the Gulf of Mexico (Andrade-Canto et al., 2013) but also in many other areas all around the world (Prants et al., 2014).

\subsection{Drifters}

Surface drifter tracks are obtained from data distributed by the Global Drifters Program (GDP), from the National Oceanic and Atmospheric Administration (NOAA) and Atlantic Oceanographic and Meteorological Laboratory (AOML) (http://www.aoml.noaa.gov/phod/dac/index. php) for the time period and regions corresponding to our simulations that we carry out with HYCOM and AVISO data. Each drifter has an identification number having five digits, which allows for the identification of different drifters in our simulations. The sampling of the drifter position is once every 6 hours.

\section{The Gulf of Mexico}

Our focus is the study of the Gulf of Mexico, in the area between latitudes $22-28^{\circ} \mathrm{N}$ and longitudes $80-91^{\circ} \mathrm{W}$, from 11 June through July to 7 August in the year 2010. This choice corresponds to the months just after the Deepwater Horizon oil spill in the year 2010, although this work does not aim to achieve any conclusion regarding those events.

The Yucatán-Florida current system is in this region, also known as the Loop Current (Forristall et al., 1992; AndradeCanto et al., 2013; Sulman et al., 2013). This current enters the gulf through the Yucatán channel, then circulates clock- wise and exits the gulf through the Straits of Florida, flowing eventually into the Gulf Stream. During the period under consideration, our data show that this current is pinched off forming first a large anticyclonic loop, which survives until 24 June. After this date, the big loop breaks into several eddies, which become weaker and weaker by the end of the period of interest.

Our study attempts to understand the consistency between the evolution of drifters during this period and the Lagrangian features provided by the two data sets. In particular, agreement in the following aspects will be analyzed: the role played by manifolds that represent barriers to transport, drifter dispersion produced by hyperbolic trajectories, drifter confinement produced by eddies, and material ejection through lobe dynamics.

\section{The dynamical system}

We are interested in the study of transport due solely to advection. In this case a particle evolves according to

$\frac{\mathrm{d} \boldsymbol{x}}{\mathrm{d} t}=\boldsymbol{v}(\boldsymbol{x}, t), \boldsymbol{x} \in \mathbb{R}^{n}, t \in \mathbb{R}$.

In our geophysical applications, we consider twodimensional motion of particles, i.e., $n=2$. We assume that $\boldsymbol{v}(\boldsymbol{x}, t)$ is $C^{r}(r \geq 1)$ in $\boldsymbol{x}$ and continuous in $t$. In our study, the velocity field $\boldsymbol{v}$ given in Eq. (1) is defined as a data set, provided by observations or simulations and has been described in the previous section.

The equations of motion that describe the horizontal evolution of particle trajectories on a sphere are

$$
\begin{aligned}
\frac{\mathrm{d} \phi}{\mathrm{d} t} & =\frac{u(\phi, \lambda, t)}{R \cos (\lambda)}, \\
\frac{\mathrm{d} \lambda}{\mathrm{d} t} & =\frac{v(\phi, \lambda, t)}{R} .
\end{aligned}
$$

Here the variables $(\phi, \lambda)$ are longitude and latitude; $u$ and $v$, respectively, represent the eastward and northward components of the velocity field. The particle trajectories must be integrated using Eqs. (2)-(3); since information is provided solely on a discrete space-time grid, the first issue to deal with is that of interpolation. We have daily or hourly maps of the velocity field, and these are too coarse of a time grid to provide a time step for the integration of particle trajectories. However, this frequency sampling is adequate in the sense that changes of the velocity field below that resolution are smooth enough to be adequately captured by an interpolator. Days are a typical timescale for the system (Eqs. 23 ), and this is the unit of time in which results are reported. A recent paper by Mancho et al. (2006a) compares different interpolation techniques for tracking particle trajectories. Bicubic interpolation in space (Press et al., 1999) and thirdorder Lagrange polynomials in time are shown to provide a 
computationally efficient and accurate method. In our calculations we replace the bicubic spatial interpolation in space by the spline interpolation supplied by MATLAB as we have verified that it provides similar accuracy.

\section{Lagrangian descriptors}

Solutions of dynamical systems are qualitatively described according to Poincaré's idea to seek geometrical structures in the phase space that can be used to organize trajectories schematically into distinct regions corresponding to qualitatively different types of trajectories.

In time-independent systems, i.e., those for which Eq. (1) does not depend explicitly on time, fixed points are essential for describing the solutions geometrically. This is so because stable and unstable manifolds of hyperbolic fixed points act as separatrices that divide the phase portrait into regions in which particles have different dynamical fates. To achieve this geometrical representation in time-dependent aperiodic dynamical systems, special hyperbolic trajectories have been distinguished, which have been called distinguished hyperbolic trajectories (Ide et al., 2002; Madrid and Mancho, 2009), and the stable and unstable manifolds of such trajectories have been successfully used to describe transport in geophysical flows (Mancho et al., 2008; Branicki et al., 2011; Mendoza et al., 2010).

In this work our geometrical representation is built with Lagrangian descriptors (LDs). LDs are based on a function referred to as $M$ that was proposed in Madrid and Mancho (2009) as a building block of the definition of distinguished trajectories. Later in Mendoza and Mancho (2010), this function was introduced as a Lagrangian descriptor, i.e., a function that provides a global dynamical picture of the geometrical structures governing transport in arbitrary timedependent flows. Lagrangian descriptors were extended and theoretically justified in simple examples in Mancho et al. (2013) and Mendoza and Mancho (2012). In this article we will focus on the first approach to LDs given by a function $M$ that measures the Euclidean arc length of the curve outlined by a trajectory passing through $\boldsymbol{x}^{*}$ at time $t^{*}$. The trajectory is integrated from $t^{*}-\tau$ to $t^{*}+\tau$. This is mathematically expressed as follows: for all initial conditions $\boldsymbol{x}^{*}$ in an open set $\mathcal{B} \in \mathbb{R}^{n}$, at a given time $t^{*}$, the Lagrangian descriptor is a function $M\left(x^{*}, t^{*}\right)_{v, \tau}:(\mathcal{B}, t) \rightarrow \mathbb{R}$ given by

$$
M\left(\boldsymbol{x}^{*}, t^{*}\right)_{v, \tau}=\int_{t^{*}-\tau}^{t^{*}+\tau} \sqrt{\sum_{i=1}^{n}\left(\frac{\mathrm{d} x_{i}(t)}{\mathrm{d} t}\right)^{2}} \mathrm{~d} t .
$$

Here $x_{1}(t), x_{2}(t), \ldots, x_{n}(t)$ are the components in $\mathbb{R}^{n}$ of a trajectory $\boldsymbol{x}(t)$. The function $M$ is defined for dynamical systems in arbitrary dimension $n$, but for our current application we will take $n=2$. It depends on $\tau$ and also, of course, on the velocity field $\boldsymbol{v}$.
For small $\tau$ the output provided by $M$ is smooth, almost without structure and resembling that of Eulerian currents. This is confirmed from Fig. 1a, which shows the evaluation of $M$ on AVISO in the Loop Current region in the Gulf of Mexico for $\tau=3$ days. Figure $1 \mathrm{~b}$ and c confirm that for increasing $\tau$ the function $M$ loses regularity along lines, which, from the numerical point of view, have been systematically confirmed to be aligned with the stable and unstable manifolds of hyperbolic trajectories (Mendoza and Mancho, 2010; Mendoza et al., 2010; de la Cámara et al., 2012, 2013; Mancho et al., 2013). In Fig. 1b the lines aligned with the stable and unstable manifolds of a hyperbolic point are clearly seen, and further details are distinguished in Fig. 1c. The Lagrangian features are not provided by the specific values taken by $M(M$ has length dimension and the units are those of the plane on which the integration of the trajectory is performed - see Eqs. 2-3), but the Lagrangian information is contained in the structure of $M$; for this reason the color scale in the figures is not included. To make an interpretation of $M$, it is sufficient to know that the red color expresses the maximum arc-length values, obtained for particles moving rapidly, as they are placed in jets, and blue color are minimum arc-length values obtained in regions where particles move calmly.

There is no a priori knowledge on how long the time integration interval $\tau$ should be taken in order to highlight Lagrangian structures. It has been observed that it depends on the data set under study. On the other hand, the analytical arguments given for a simple example in Mancho et al. (2013) suggest that, in hyperbolic regions with large expansion or contraction rates, the formation of these singular features could be achieved at shorter $\tau$. Figure 1 suggests $\tau=15$ days as an appropriate choice for the AVISO data.

The increasing complexity of $M$ versus $\tau$ is expected from the nature of $M$, since it is reflecting the history of initial conditions on open sets, and in highly chaotic systems this history is expected to be more tangled for longer time intervals. Thus $\tau$ values larger than 15 days could have been taken, which would have made visible many details of the manifold structure, as reported for instance in Mendoza and Mancho (2010) and Mancho et al. (2013). This is consistent with what is obtained from the direct computation of manifolds (Mancho et al., 2003, 2004) for which the longer the integration time is, the larger the pieces of manifolds are. At the moment there exist no formal proof that guarantees that these singular features are truly advected by the flow, although some analytical arguments are discussed in this respect in Mancho et al. (2013).

Figure 2 shows the output on the same region for the same $\tau$ selection on the HYCOM data. Again it is observed that for increasing $\tau$ more and more Lagrangian structure is observed. The position of the hyperbolic point here is shifted with respect to that observed in the AVISO data. Also the manifold structure for a given $\tau$ is much richer than in the AVISO data. In fact for $\tau=15$ the mesoscale structure is 
Jun 12 12:00
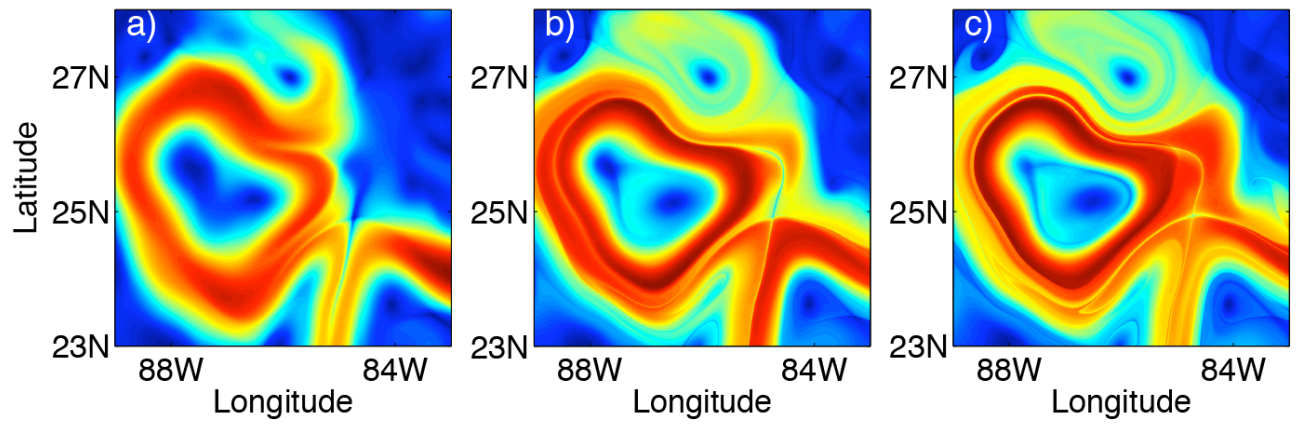

Figure 1. Evaluation of the $M$ function in the Gulf of Mexico for AVISO data. (a) $\tau=3$ days; (b) $\tau=9$ days; (c) $\tau=15$ days. The position of the manifolds is highlighted in this figure.

Jun 12 12:00
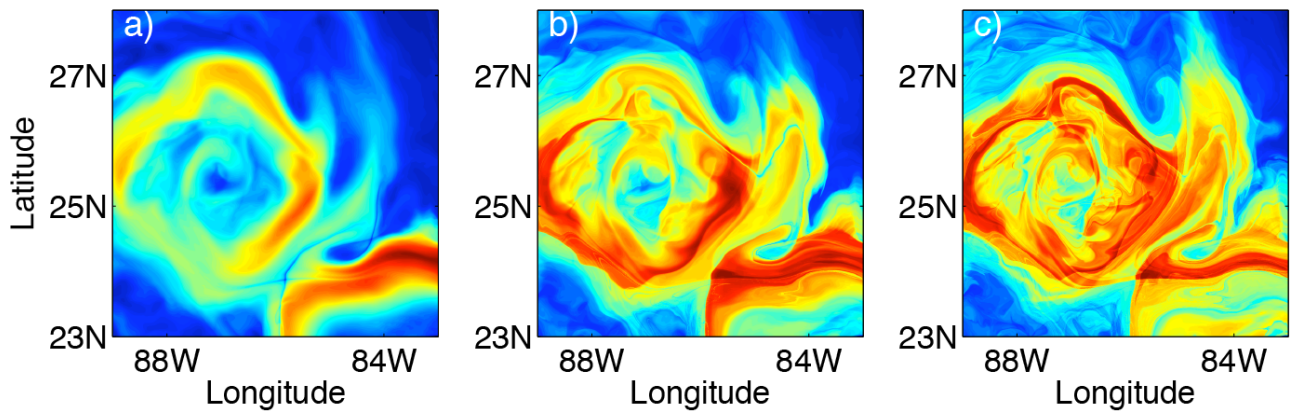

Figure 2. Evaluation of the $M$ function in the Gulf of Mexico for HYCOM data. (a) $\tau=3$ days; (b) $\tau=9$ days; (c) $\tau=15$ days.

rather blurred when compared to $\tau=9$. This confirms that the appropriate $\tau$, which allows the manifold visualization, depends on the data. In the following sections we will produce $M$ maps with $\tau=9$ days for the HYCOM data as that is enough to highlight the mesoscale structure.

The absence of regularity of $M$ at large $\tau$ highlights the position of invariant manifolds. These contrasts correspond to transport barriers as confirmed in Fig. 3. The direct computation of the stable and unstable manifolds is displayed side by side with the structure of $M$, and the coincidence is evident. This calculation is done following the method developed by Mancho et al. (2003, 2004, 2006b) and Mendoza and Mancho (2012), and as explained this is done by integrating particle trajectories. In particular, stable and unstable manifolds are, respectively, composed of particle trajectories that at a given time are placed in a small segment along the stable or unstable subspaces of a given distinguished hyperbolic trajectory (DHT). Manifold structures displayed in Fig. 3c have been obtained after integrating backwards and forwards in time using AVISO data for a period of 15 days, and those in Fig. 3d have been obtained after integrating backwards and forwards in time with HYCOM data for a period of 9 days. The direct computation of manifolds displays a less complex appearance than those obtained from the $M$ function because, as noted in Mendoza and Mancho (2012), it is done for selected DHTs that may leave out many other DHTs in the neighborhood, while $M$ accounts for all all stable and unstable manifolds from all possible DHTs in the vicinity.

The presence of a rich manifold structure for HYCOM data when compared to AVISO is due to the presence of small "noisy" structures in the velocity fields themselves for the HYCOM output, which is absent in AVISO. "Noisy" structures in the HYCOM velocity field are possible due to the smaller grid size used in this case. However, below the resolution grid these "noisy" structures are assumed to follow the bicubic interpolation, which is smooth within each cell and $C^{1}$ at boundaries of the grid. Figure 4 confirms this point. In this figure we show the eastwards $u$ and northwards $v$ velocity components along the longitude $86^{\circ} \mathrm{W}$ on 12 June 2010 . The AVISO velocity (in blue) is smoother than the HYCOM velocity (in black).

Hernández-Carrasco et al. (2011) have discussed the reliability and dependence on the grid size of a Lagrangian diagnosis based on FSLE. In particular they have considered two types of grid sizes. One of the sizes is related to the grid in which the velocity field is given (called the V-grid). This grid is also present in our case. Changing the size here is related to introducing slight modifications in the underlying velocity 

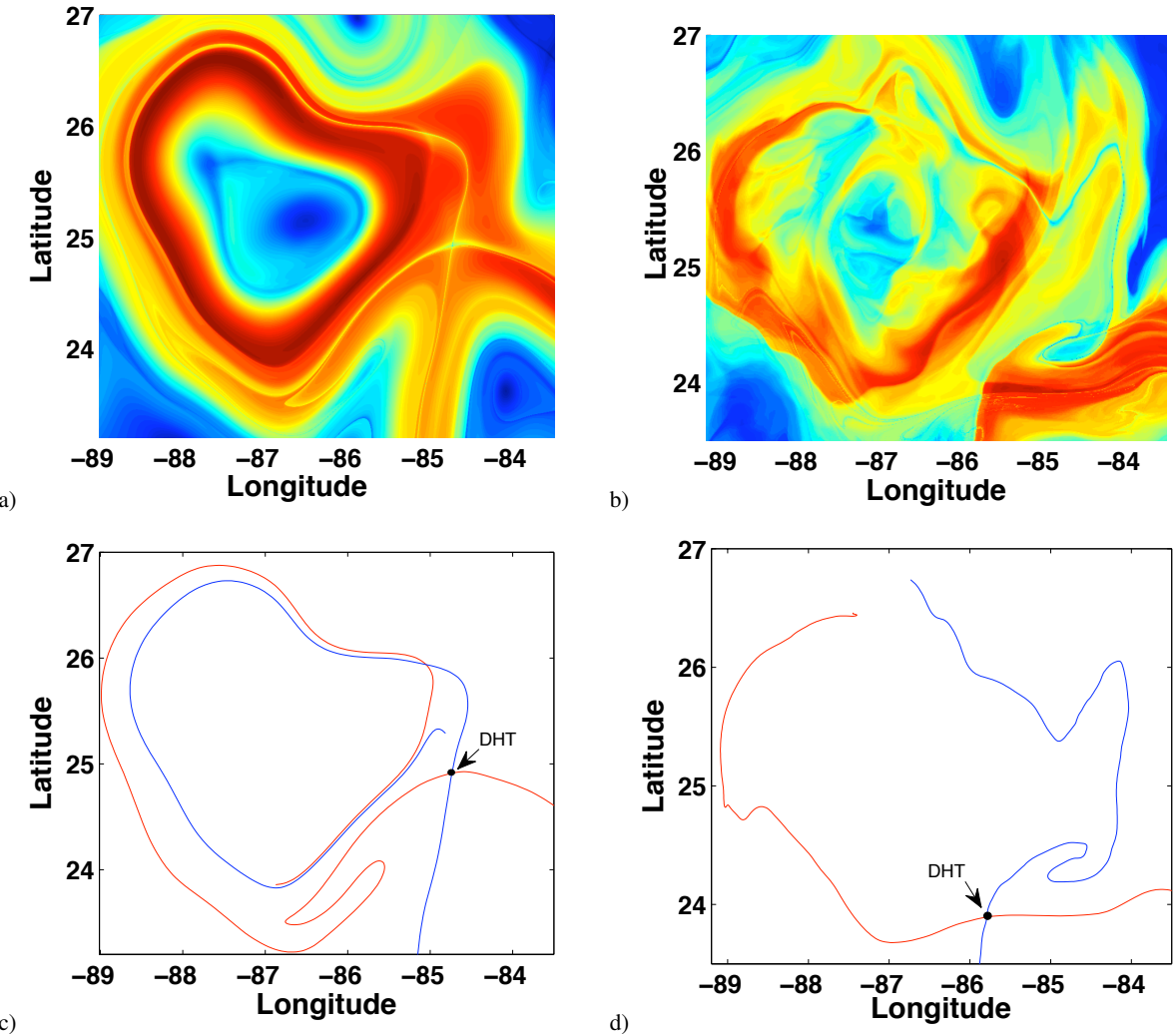

Figure 3. Evaluation of the $M$ function and visualization of the manifold structure on 12 June 2010 at 12:00 UTC. (a) Evaluation of $M$ with AVISO for $\tau=15$ days; (b) $M$ on HYCOM for $\tau=9$ days; (c) manifolds with AVISO computed for 15 days; (d) manifolds with HYCOM computed for 9 days.
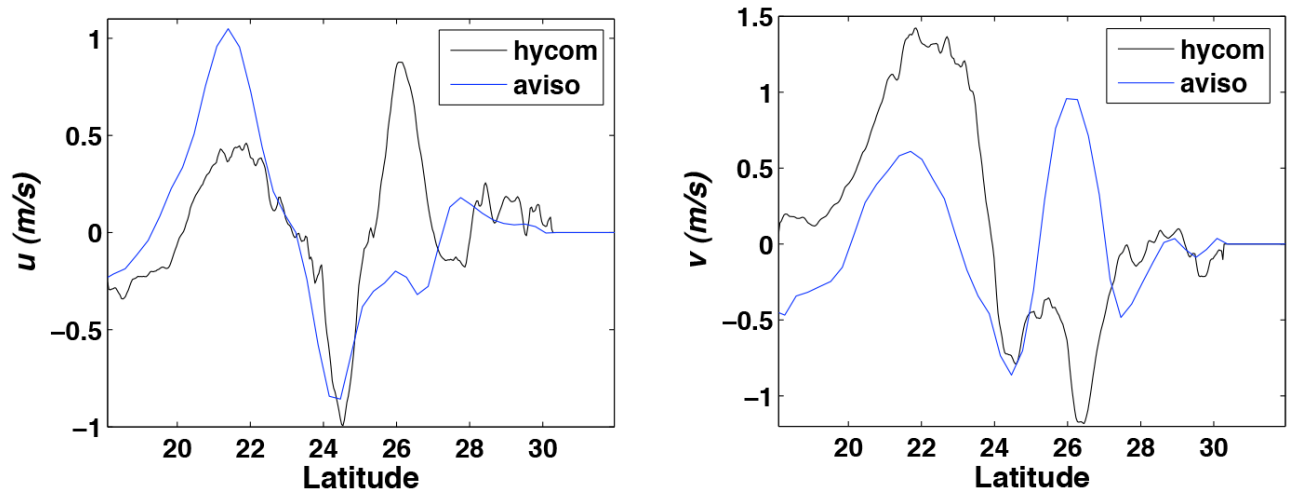

Figure 4. Velocity components along the longitude $86^{\circ} \mathrm{W}$ for HYCOM (black) and AVISO (blue) data on 12 June 2010: (a) eastwards velocity component and (b) northwards velocity component.

field defining the system (Eqs. 2-3), as this continuous dynamical system is produced from an interpolation that is not identical for each grid choice. Lagrangian structures are expected to be robust versus slight perturbations to the velocity field (see for instance Haller, 2002) given that chaotic saddles are structurally stable objects (although this has not been proven for aperiodically time-dependent velocity fields, it is a reasonable conjecture). Consistently Lagrangian descrip- tors have been shown to produce similar outputs for slightly modified velocity fields (Mendoza and Mancho, 2010, 2012). A second grid size effect discussed by Hernández-Carrasco et al. (2011) - the F-grid - is related to the adjustment of a parameter, $\delta$, that measures the initial separation of trajectories on which FSLEs depend. However, this does not influence our results as LDs do not depend on such a parameter. 

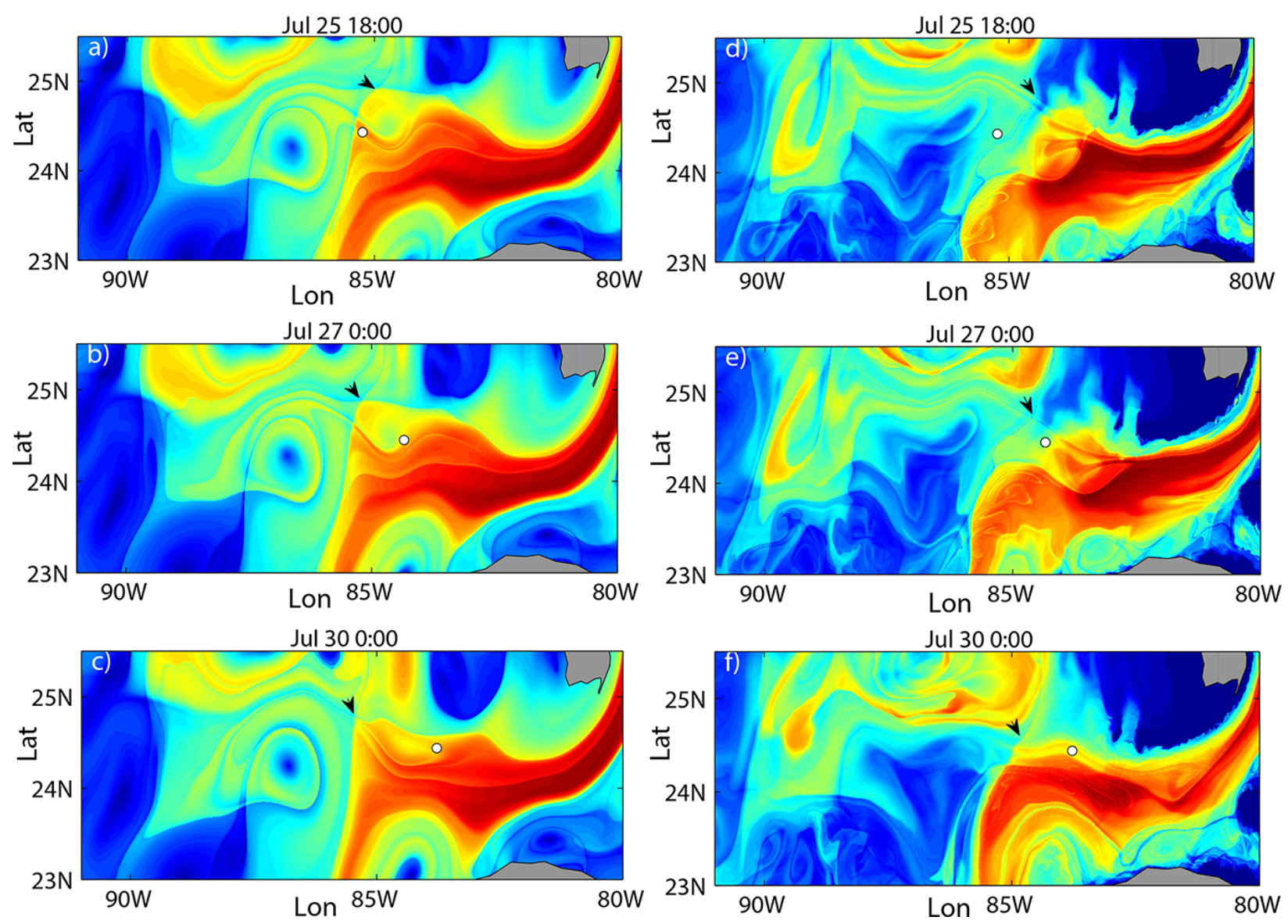

Figure 5. Sequence showing the evolution of the Lagrangian structures with AVISO data from (a) to (c) and with HYCOM data and from (d) to (f) for 25, 27 and 30 July respectively. Time is given as Universal Coordinated Time (UTC). The drifter "98945" is overlapped with these structures. It is observed how it is injected within the Gulf Stream through a lobe. The position of the hyperbolic trajectory is highlighted in each frame with the arrow.

Mancho et al. (2013) have shown, in the context of simple and controlled analytical examples, that Lagrangian descriptors present numerous advantages versus other Lagrangian tools common in the literature such as FTLEs or finite time averages. Rempel et al. (2013) have extended the comparison with FTLE to the context of 3-D turbulent flows. Some of the advantages found are listed next; LDs are computationally cheaper than FTLEs; on data sets the function $M$ is less sensitive to the quality of the interpolation of the vector field. LDs have not been reported to produce ghost structures (i.e., false Lagrangian patterns), while there exists substantial literature reporting these issues for FTLE (Branicki and Wiggins, 2010; Farazmand and Haller, 2012; Rempel et al., 2013; Mancho et al., 2013). LDs are easier to program because they do not require the adjustment of any parameter except $\tau$, which is directly related to the detail required in the manifolds. The maps of LDs systematically display sharper plots that facilitate the identification of Lagrangian coherent structures and lobe dynamics. For instance de la Cámara et al. (2012) identify routes of transport that cross the Antarctic polar jet. This has not been possible to obtain from FTLEs since they provide a blurred pattern, full of spurious structures. De la Cámara et al. (2013) provide a picture of the Antarctic polar jet that finds Rossby wave breaking evidence in the interior of the vortex, while these features have been unnoticed with other Lagrangian approaches. All these reasons justify our choice.

\section{Results}

In this section we describe the ocean Lagrangian skeleton and correlate it with the paths of several drifters in the Gulf of Mexico during the period under study. We compare side by side the outputs obtained from the HYCOM model and the AVISO data sets. At this point, it is not always clear which data set is more representative of the state of the ocean. Our analysis methodology is based on assuming that consistency between different data sources is a good metric for data quality. In particular, a sign of consistency is the agreement between the Lagrangian features and the paths followed by the 

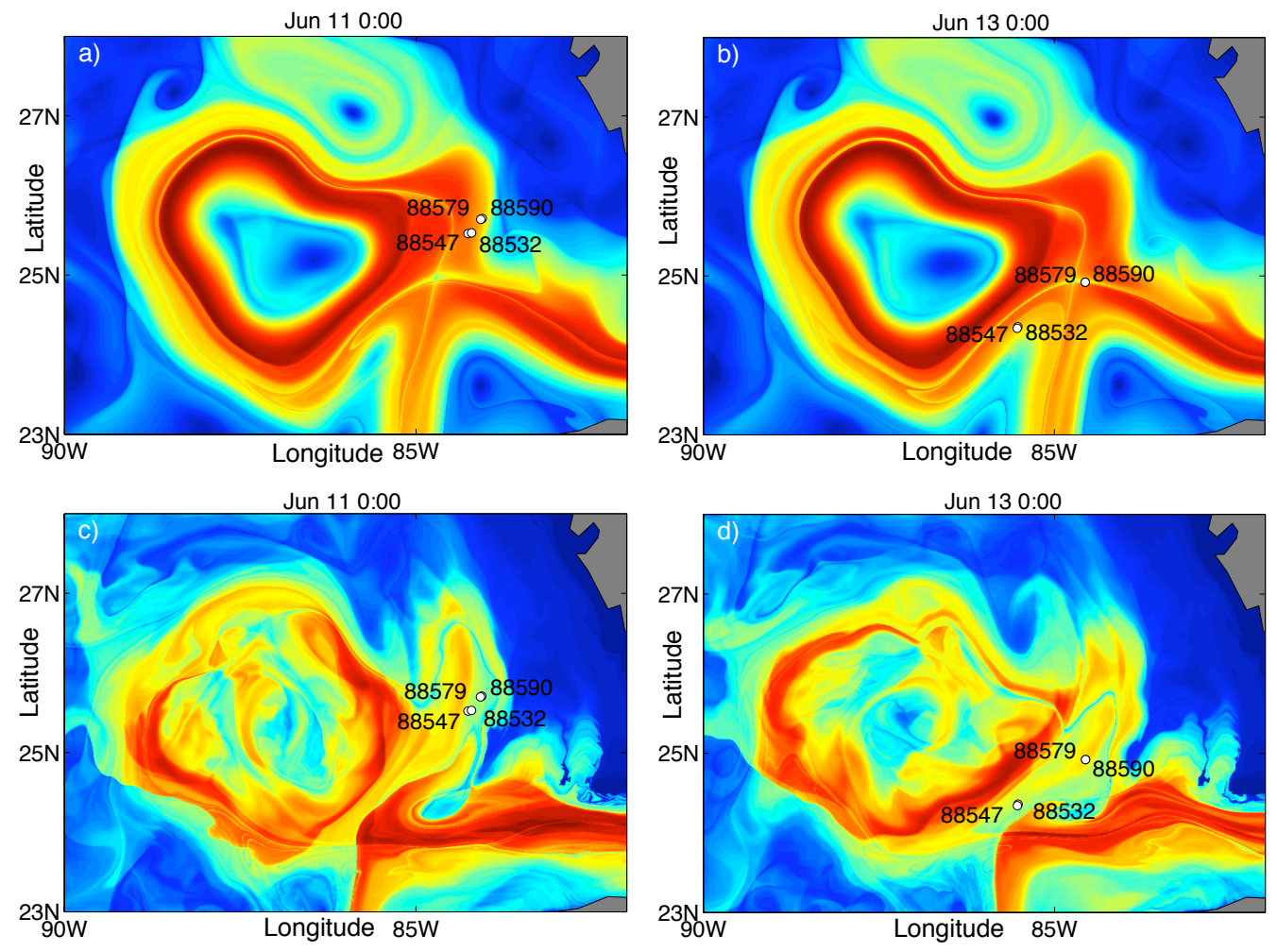

Figure 6. Sequence that shows the evolution of the Lagrangian structures with AVISO - (a) and (b) - and HYCOM - (c) and (d) - data. Drifters " 88532 ", "88547", "88590", and " 88579 " are overlapped with these structures. The left column displays results for 11 June and the right one for 13 June.

drifters. Our focus is on this type of consistency because a comparison of individual trajectories of in situ drifters with the corresponding simulated trajectories of the respective velocities is not going to be close. This something expected in a chaotic system and has been reported for instance by Mancho et al. (2006a) and de la Cámara et al. (2010). Despite the fact that individual trajectories do not evolve close to each other, the underlying Lagrangian skeleton can be close or similar. Chaotic saddles are structurally stable objects and thus Lagrangian structures should be robust versus slight perturbations to the velocity field. For these reasons we base our analysis on Lagrangian features and not on individual trajectories.

During the period of interest, 36 drifters were tracked in the region of study. Our focus is on analyzing the agreement on typical dynamical features in the velocity field and the drifter motion. In the period under study, some drifters leave the domain under consideration rapidly, which is consistent with the observed behavior of the Gulf Stream. This is so in both HYCOM and AVISO data. In particular drifters 98944, 98934, 98942, 98904, 98936, 98941, 98910, and 98945 follow this fate. Figure 5 shows how the drifter 98945 is injected into the main stream through a dynamical object referred to as a "lobe". Lobes are water masses surrounded by a piece of a stable manifold and a piece of an unstable manifold. Close to hyperbolic points, lobes evolve forming filaments as described for instance in Mancho et al. (2008). This elongating structure is observed in Fig. 5 both for HYCOM and AVISO with the drifter inside it while it is swept along the current. The presence of the hyperbolic point is highlighted in the sequence.

On the first day of our study, 11 June, the Loop Current is pinched off forming a large anticyclonic Loop as reported by Sulman et al. (2013). Consistently, this scenario is observed both from AVISO and HYCOM data as observed from Fig. 6. It is noted though that the position of the main hyperbolic point is different at each data set. Figure 6 shows that the evolution of drifters $88532,88547,88590$, and 88579 is consistent with the location of the hyperbolic trajectory in HYCOM but not in AVISO. In this latter case if drifters had followed the Lagrangian features highlighted in the figure, they should have evolved bending towards the east without crossing the invariant manifolds that intersect at that position. This inconsistency is not observed in the HYCOM data as the hyperbolic trajectory is shifted towards the southeast. Other examples exist during the period under study that confirm the efficiency of HYCOM data to correctly position the hyperbolic trajectory in a manner that justifies drifter's dispersion. 

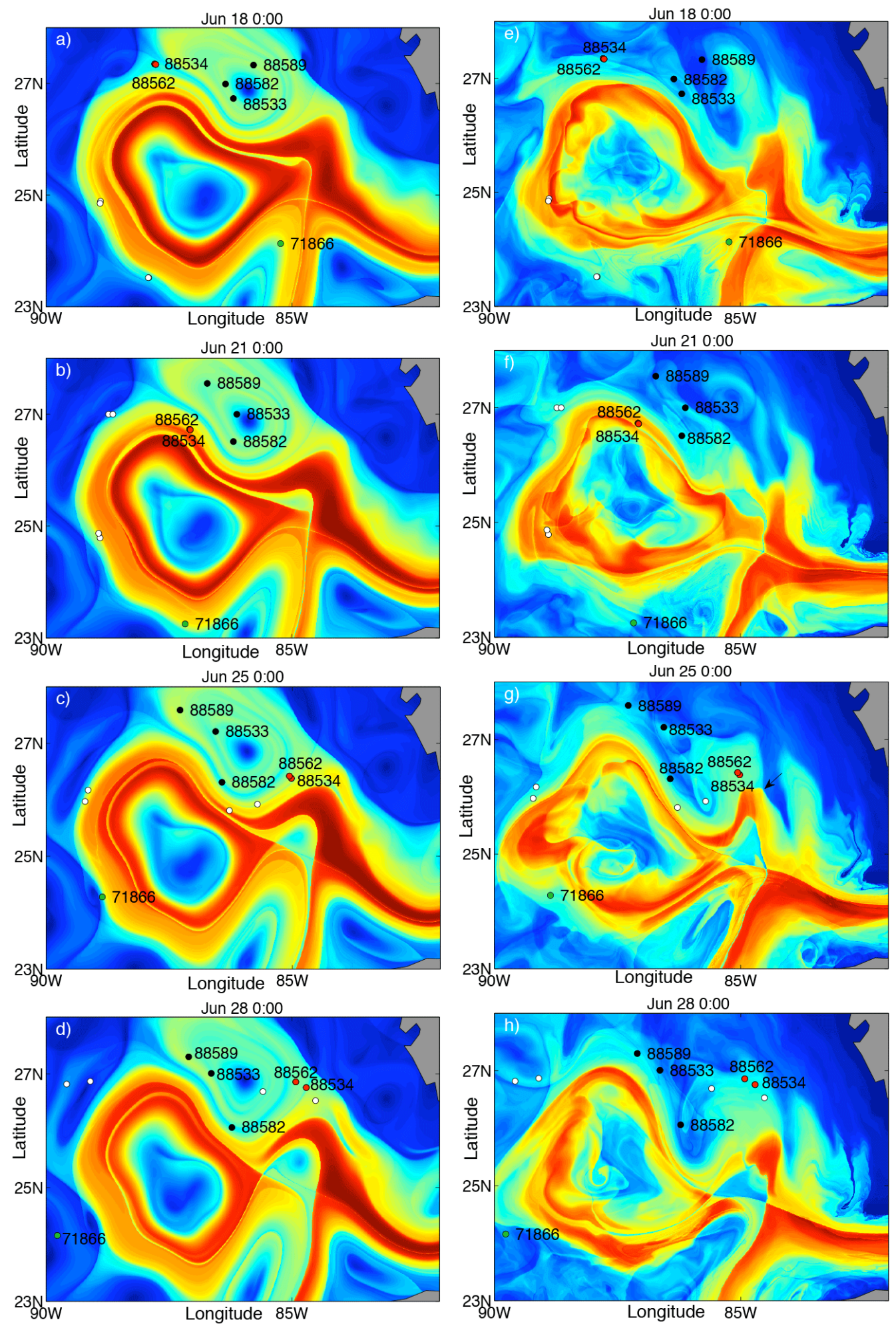

Figure 7. Sequence that shows the evolution of the Lagrangian structures with AVISO - (a) and (d) - and HYCOM - (e) and (h) - data. Drifters "88532", "88547”, "88590", "88579", already depicted in Fig. 6, are represented without labels and in white as before. Drifters "88562" and "88534" are overlapped in red and drifter "71866" in green. Drifters "88589", "88533" and "88582" are in black. The first row displays results for 18 June, the second for 21 June, the third row for 25 June and the fourth row for 28 June. An arrow highlights the position of a hyperbolic trajectory in (g). 

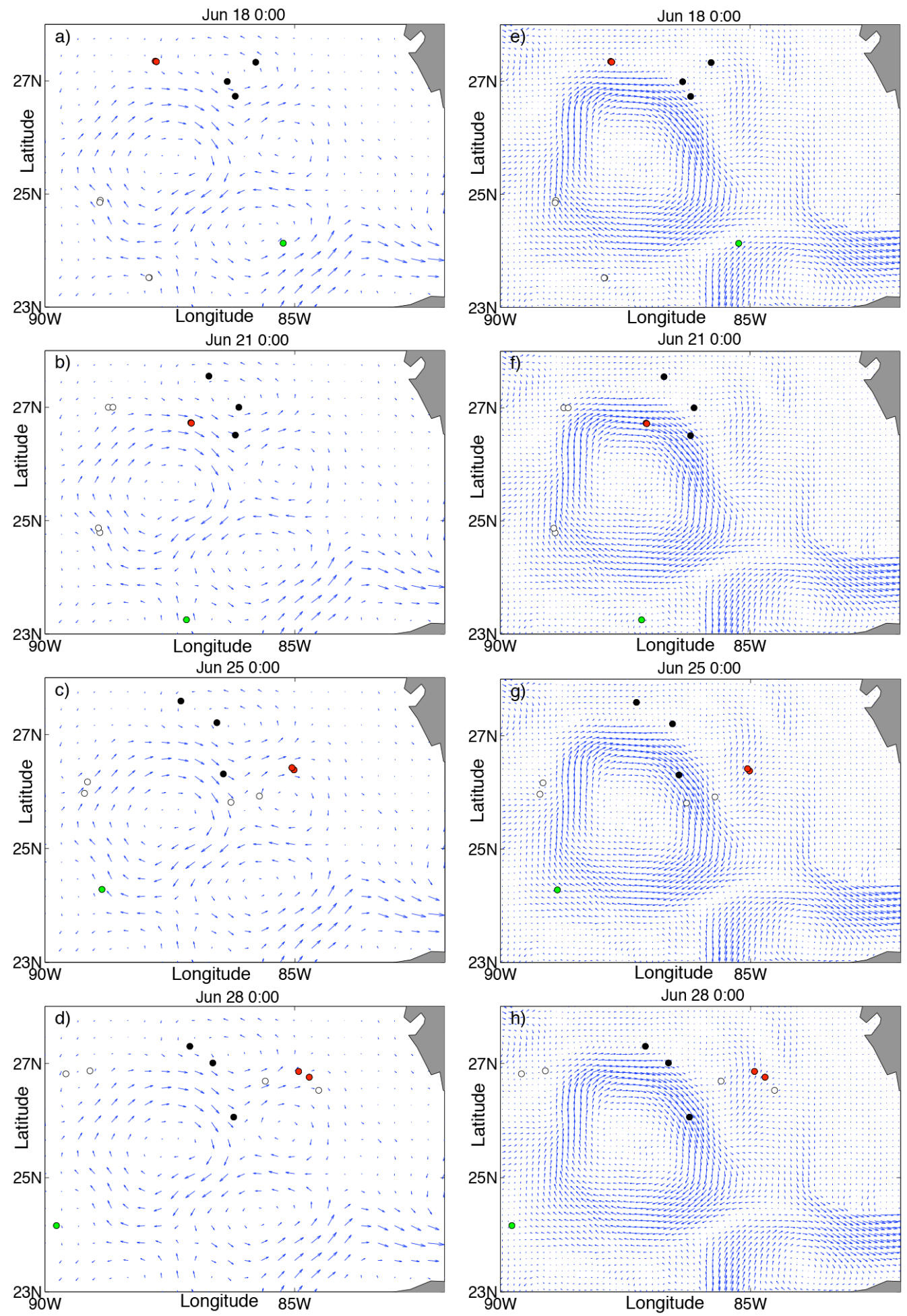

Figure 8. Sequence that shows the evolution of the Eulerian velocities from AVISO data from (a) to (d) and from HYCOM data from (e) to (h). Drifters of Fig. 7 are depicted with the same color code but without identifiers. It is hard to understand drifter evolution directly from these Eulerian fields. 
The above drifters, together with drifter 71866 , that joins the Loop Current from the south, circulate clockwise in the stream consistently with the pictures provided both by HYCOM and AVISO. This is confirmed in Fig. 7. Drifters leave the loop, although without showing a clear connection with hyperbolic trajectories or lobes. This is so both for HYCOM and AVISO data.

The Loop Current, as explained in Sect. 3 and visible in Fig. 7, breaks into smaller eddies after 24 June in the HYCOM simulations and around 25 June in AVISO. The number of eddies though is not the same for both data. In particular at the northern part of the loop, AVISO displays clear eddy-like structures, while HYCOM does not. Figure 7 confirms that the evolution of drifters 88562 and 88534 from 18 June through 28 June is consistent with Lagrangian structures from both data, although Lagrangian structures are different in each case. Figure 7 shows four snapshots from AVISO and HYCOM for these drifters. They are trapped into the loop, and their position is displayed on 18 June, They evolve in both cases close to a manifold highlighted in the middle of the jet. On 25 June the drifter in AVISO seems to be trapped in an upper eddy, while HYCOM suggests that the drifter evolves within a lobe that elongates due to the presence of a hyperbolic point marked in the figure, which could cause the separation between drifters. Eventually, the position on 28 June is marked. Drifters are clearly within an eddy in the AVISO data, and this structure is unobserved in HYCOM.

The Lagrangian descriptors truly help to make an interpretation of the dynamics. Figure 8 shows the Eulerian fields that correspond to the same sequence of snapshots as seen in Fig. 7. The AVISO data are not provided in hourly intervals as is the case of the HYCOM data; thus what we show in this figure is the interpolation in time on the grid nodes of the velocity field supplied on a daily basis. First is observed that from the Eulerian point of view both the HYCOM model and AVISO present a similar structure, but the HYCOM model has a much higher resolution. From these pictures the perception of the eddy structures is much weaker, and the hyperbolic trajectories are not distinguished.

Many drifters, such as $8858988582,88533,98905,98938$, 98909, 98914, 98945, 98916, 98915, 98911, and 98939 (see some of them in black in Fig. 7), follow approximate circular motions that seem consistently linked to eddies observed in AVISO data. However, there exist events for which the Lagrangian skeleton that connects the motions of drifters passing form one eddy to another is obscure. Sometimes it happens that the data do not provide clear Lagrangian patterns that justify these transactions. Mesoscale eddies seem to be more faded on HYCOM, as a lot of Lagrangian submesoscale structures distort them. However, this does not imply that the the motion of the drifters is inconsistent in this case.

From the point of view taken in this article, inconsistencies would indicate that the velocity field deduced from the altimetry or from mathematical models, despite being ac- curate for describing some events, may be not be precise enough for describing all of them. On the other hand, inconsistencies could be caused by drifters that are not truly Lagrangian tracers (Tallapragada and Ross, 2008), and thus their dynamics would not be forced to follow that of authentic Lagrangian tracers. We note however that this is not the perspective adopted in most of the Lagrangian studies in oceanic contexts (Griffa et al., 2013), in which drifter trajectories are even assimilated into the model considering them as the "truth" (Nencioli et al., 2011). A thorough revision of these questions is of great interest but is beyond the scope of this article.

\section{Summary, conclusions, and outlook}

In this article we have explored the ability of Lagrangian tools to extract information from oceanic data and their role in helping to interpret drifter paths on the ocean surface. We have found that both AVISO and HYCOM data do a reasonable job in revealing the Lagrangian mesoscale structures that govern the drifter motion.

The Lagrangian analysis is shown to be very useful in order to make an interpretation of drifter routes and highlighting differences between HYCOM and AVISO. The velocity fields are all rather "similar" based on an Eulerian comparison (i.e., just comparing the velocity fields). But they are different, based on Lagrangian diagnostics and the hidden structures, not displayed in the Eulerian fields, truly organize the trajectories and help to characterize the quality of the data, and their inaccuracies.

At a first look AVISO and HYCOM data are different in that HYCOM has a higher resolution and presents a noisier structure, which is also reflected in the Lagrangian patterns. AVISO tends to provide clean eddy-like structures, which in HYCOM are faded by overlapped features, though consistency with respect to drifter motions seems to be present in both cases. Both AVISO and HYCOM do well in providing Lagrangian structures that guide drifter motions, as for instance within the Gulf Stream or circular motions along the Loop Current. However, evidence is provided in which AVISO fails to correctly locate hyperbolic trajectories, while in HYCOM hyperbolic trajectories are consistent with drifters trajectories.

Acknowledgements. The research of C. Mendoza and A. M. Mancho is supported by MINECO grant MTM2011-26696. The research of S. Wiggins was supported by ONR grant no. N0001401-1-0769. We acknowledge support from MINECO: ICMAT Severo Ochoa project SEV-2011-0087. Thanks are owed to CESGA, UPM and ICMAT for computing facilities.

Edited by: C. López

Reviewed by: two anonymous referees 


\section{References}

Andrade-Canto, F., Sheinbaum, J., and Zavala Sansón, L.: A Lagrangian approach to the Loop Current eddy separation, Nonlin. Processes Geophys., 20, 85-96, doi:10.5194/npg-20-85-2013, 2013.

Beron-Vera, F. J., Olascoaga, M. J., and Goni, G. J.: Surface Ocean Mixing Inferred from Different Multisatellite Altimetry Measurements, J. Phys. Oceanogr., 40, 2466-2480, 2010.

Bettencort, J. H., López, C., and Hernández-García, E.: Oceanic three-dimensional Lagrangian coherent structures: A study of a mesoscale eddy in the Benguela upwelling region, Ocean Modell., 51, 73-83, doi:10.1016/j.ocemod.2012.04.004, 2012.

Bleck, R.: An oceanic general circulation model framed in hybrid isopycnic-Cartesian coordinates, Ocean Modell., 4, 55-88, 2002.

Branicki, M. and Kirwan, A. D., Jr.: Stirring: The Eckart paradigm revisited, Int. J. Eng. Sci., 48, 1027-1042, 2010.

Branicki, M. and Wiggins, S.: An adaptive method for computing invariant manifolds in non-autonomous, three-dimensional dynamical systems, Physica D, 238, 1625-1657, 2009.

Branicki, M. and Wiggins, S.: Finite-time Lagrangian transport analysis: stable and unstable manifolds of hyperbolic trajectories and finite-time Lyapunov exponents, Nonlin. Processes Geophys., 17, 1-36, doi:10.5194/npg-17-1-2010, 2010.

Branicki, M., Mancho, A. M., and Wiggins, S.: A Lagrangian description of transport associated with a Front-Eddy interaction: application to data from the North-Western Mediterranean Sea, Physica D, 240, 282-304, doi:10.1016/j.physd.2010.09.003, 2011.

Chassignet, E. P., Hurlburt, H. E., Smedstad, O. M., Halliwell, G. R., Hogan, P. J., Wallcraft, A. J., Baraille, R., and Bleck, R.: The HYCOM (HYbrid Coordinate Ocean Model) data assimilative system, J. Mar. Syst.,65, 60-83, doi:10.1016/j.jmarsys.2005.09.016, 2007.

de la Cámara, A., Mechoso, C. R., Ide, K., Walterscheid, R., and Schubert, G.: Polar night vortex breakdown and large-scale stirring in the southern stratosphere, Clim. Dynam., 35, 965-975, 2010.

de la Cámara, A., Mancho, A. M., Ide, K., Serrano, E., and Mechoso, C.: Routes of transport across the Antarctic polar vortex in the southern spring., J. Atmos. Sci., 69, 753-767, doi:10.1175/JAS-D-11-0142.1, 2012.

de la Cámara, A., Mechoso, R., Mancho, A. M., Serrano, E., and Ide., K.: Quasi-horizontal transport within the Antarctic polar night vortex: Rossby wave breaking evidence and Lagrangian structures., J. Atmos. Sci., 70, 2982-3001, doi:10.1175/JAS-D12-0274.1, 2013.

d'Ovidio, F., Fernández, V., Hernández-García, E., and López, C.: Mixing structures in the Mediterranean sea from finitesize Lyapunov exponents, Geophys. Res. Lett., 31, L12203, doi:10.1029/2004GL020328, 2004.

Farazmand, M. and Haller, G.: Computing Lagrangian Coherent Structures from variational theory, Chaos, 22, 013128, doi:10.1063/1.3690153, 2012.

Forristall, G. Z., Schaudt, K. J., and Cooper, C. K.: Evolution and Kinematics of a Loop Current Eddy in the Gulf of Mexico During 1985, J. Geophys. Res., 97, 2173-2184, 1992.

Froyland, G., Horenkamp, C., Rossi, V., Santitissadeekorn, N., and Gupta, A. S.: Three-dimensional characterization and tracking of an Agulhas Ring, Ocean Modell., 52-53, 69-75, 2012.
Griffa, A., Haza, A., Özgökmen, T. M., Molcard, A., Taillandier, V., Schroeder, K., Chang, Y., and Poulain, P. M.: Investigating transportpathwaysintheocean, Deep Sea Res. II, 85, 81-95, 2013.

Haller, G.: Finding finite-time invariant manifolds in twodimensional velocity fields, Chaos, 10, 99-108, 2000.

Haller, G.: Distinguished material surfaces and coherent structure in three-dimensional fluid flows, Physica D, 149, 248-277, $2001 \mathrm{a}$.

Haller, G.: Lagrangian structures and the rate of strain in a partition of two-dimensional turbulence, Phys. Fluids, 13, 33653385, 2001b.

Haller, G.: Lagrangian coherent structures from approximate velocity data, Phys. Fluids, 14, 1851-1861, 2002.

Haller, G. and Yuan, G.: Lagrangian coherent structures and mixing in two-dimensional turbulence, Physica D, 147, 352-370, 2000.

Haza, A. C., Griffa, A., Martin, P., Molcard, A., Özgökmen, T. M., Poje, A., Barbanti, R., Book, J., Poulain, P., Rixen, M., and Zanasca, P.: Model-based directed drifter launches in the Adriatic Sea: Results from the DART experiment, Geophys. Res. Lett., 34, L10605, doi:10.1029/2007GL029634, 2007.

Haza, A. C., Özgökmen, T. M., Griffa, A., Molcard, A., Poulain, P. M., and Peggion, G.: Transport properties in small-scale coastal flows: relative dispersion from VHF radar measurements in the Gulf of La Spezia, Ocean Dynam., 60, 861-882, 2010.

Hernández-Carrasco, I., López, C., Hernández-García, E., and Turiel, A.: How reliable are finite-size Lyapunov exponents for the assessment of ocean dynamics?, Ocean Modell., 36, 208218, 2011.

Ide, K., Small, D., and Wiggins, S.: Distinguished hyperbolic trajectories in time-dependent fluid flows: analytical and computational approach for velocity fields defined as data sets, Nonlin. Processes Geophys., 9, 237-263, doi:10.5194/npg-9-237-2002, 2002.

Jones, C. K. R. T. and Winkler, S.: Invariant manifolds and Lagrangian dynamics in the ocean and atmosphere, in: Handbook of dynamical systems, 55-92, North-Holland, Amsterdam, 2002.

Kuznetsov, L., Toner, M., Kirwan Jr., A. D., Jones, C. K. R. T., Kantha, L. H., and Choi, J.: The Loop Current and adjacent rings delineated by Lagrangian analysis of the near-surface flow, J. Mar. Res., 60, 405-429 doi:10.1357/002224002762231151, 2002.

Lekien, F., Shadden, S., and Marsden, J. E.: Lagrangian coherent structures in n-dimensional systems, J. Math. Phys., 48, 065404, doi:10.1063/1.2740025, 2007.

Madrid, J. A. J. and Mancho, A. M.: Distinguished trajectories in time dependent vector fields, Chaos, 19, 013111, doi:10.1063/1.3056050, 2009.

Malhotra, N. and Wiggins, S.: Geometric Structures, Lobe Dynamics, and Lagrangian Transport in Flows with Aperiodic TimeDependence, with Applications to Rossby Wave Flow, J. Nonlin. Sci., 8, 401-456, 1998.

Mancho, A. M., Small, D., Wiggins, S., and Ide, K.: Computation of Stable and Unstable Manifolds of Hyperbolic Trajectories in Two-Dimensional, Aperiodically Time-Dependent Vectors Fields, Physica D, 182, 188-222, doi:10.1016/S01672789(03)00152-0, 2003.

Mancho, A. M., Small, D., and Wiggins, S.: Computation of hyperbolic trajectories and their stable and unstable manifolds for oceanographic flows represented as data sets, Nonlin. Processes Geophys., 11, 17-33, doi:10.5194/npg-11-17-2004, 2004. 
Mancho, A. M., Small, D., and Wiggins, S.: A comparison of methods for interpolating chaotic flows from discrete velocity data, Comput. Fluids, 35, 416-428, doi:10.1016/j.compfluid.2005.02.003, 2006a.

Mancho, A. M., Small, D., and Wiggins, S.: A tutorial on dynamical systems concepts applied to Lagrangian transport in oceanic flows defined as finite time data sets: Theoretical and computational issues, Phys. Rep., 437, 55-124, doi:10.1016/j.physrep.2006.09.005, 2006b.

Mancho, A. M., Hernández-García, E., Small, D., Wiggins, S., and Fernández, V.: Lagrangian transport through an ocean front in the North-Western Mediterranean Sea, J. Phys. Oceanogr., 38, 1222-1237, doi:10.1175/2007JPO3677.1, 2008.

Mancho, A. M., Wiggins, S., Curbelo, J., and Mendoza, C.: Lagrangian descriptors: A Method for Revealing Phase Space Structures of General Time Dependent Dynamical Systems, Commun. Nonlinear Sci., 18, 3530-3557, doi:10.1016/j.cnsns.2013.05.002, 2013.

Mendoza, C. and Mancho, A. M.: The hidden geometry of ocean flows, Phys. Rev. Lett., 105, 038501, doi:10.1103/PhysRevLett.105.038501, 2010.

Mendoza, C. and Mancho, A. M.: Review Article: "The Lagrangian description of aperiodic flows: a case study of the Kuroshio Current", Nonlin. Processes Geophys., 19, 449-472, doi:10.5194/npg-19-449-2012, 2012.

Mendoza, C., Mancho, A. M., and Rio, M.-H.: The turnstile mechanism across the Kuroshio current: analysis of dynamics in altimeter velocity fields, Nonlin. Processes Geophys., 17, 103-111, doi:10.5194/npg-17-103-2010, 2010.

Mezic, I., Loire, S., Fonoberov, V. A., and Hogan, P. A.: A New Mixing Diagnostic and Gulf Oil Spill Movement, Science, 330, 486-489, 2010.

Miller, P. D., Jones, C. K. R. T., Rogerson, A. M., and Pratt, L. J.: Quantifying transport in numerically generated velocity fields, Physica D, 110, 105-122, 1997.

Molcard, A., Poje, A. C., and Özgökmen, T. M.: Directed drifter launch strategies for Lagrangian data assimilation using hyperbolic trajectories, Ocean Modell., 12, 268-289, 2006.

Nencioli, F., d'Ovidio, F., Dogliogi, A. M., and Petrenko, A. A.: Surface coastal circulation patterns by in-situ detection of Lagrangian coherent structures, Geophys. Res. Lett., 38, L17604, doi:10.1029/2011GL048815, 2011.

Nencioli, F., d'Ovidio, F., Dogliogi, A. M., and Petrenko, A. A.: In situ estimates of submesoscale horizontal eddy diffusivity across an ocean front, J. Geophys. Res.-Oceans, 118, 7066-7080, doi:10.1002/2013JC009252, 2013.
Olascoaga, M. J.: Isolation on the West Florida Shelf with implications for red tides and pollutant dispersal in the Gulf of Mexico, Nonlin. Processes Geophys., 17, 685-696, doi:10.5194/npg-17685-2010, 2010.

Pascual, A., Faugère, Y., Larnicol, G., and Traon, P. Y. L.: Improved description of the ocean mesoscale variability by combining four satellites altimeters, Geophys. Res. Lett., 33, L02611, doi:10.1029/2005GL024633, 2006.

Pierrehumbert, R. T.: Large-scale horizontal mixing in planetary atmospheres, Phys. Fluids A, 3, 1250-1260, 1991.

Prants, S. V., Budyansky, M. V., and Uleysky, M. Yu.: Lagrangian study of surface transport in the Kuroshio Extension area based on simulation of propagation of Fukushimaderived radionuclides, Nonlin. Processes Geophys., 21, 279-289, doi:10.5194/npg-21-279-2014, 2014.

Press, W., Teukolsky, S., Vetterling, W., and Flannery, B.: Numerical Recipes in Fortran 77, The Art of Scientific Computing, Cambridge University Press, Cambdridge, 2 Edn., 1999.

Rempel, E. L., Chian, A. C.-L., Brandenburg, A., Munuz, P. R., and Shadden, S. C.: Coherent structures and the saturation of a nonlinear dynamo, J. Fluid Mechan., 729, 309-329, 2013.

Samelson, R. and Wiggins, S.: Lagrangian Transport in Geophysical Jets and Waves: The Dynamical Systems Approach, SpringerVerlag, New York, 2006.

Shadden, S. C., Lekien, F., and Marsden, J. E.: Definition and properties of Lagrangian Coherent structures from finite-time Lyapunov exponents in two-dimensional aperiodic flows, Physica D, 212, 271-304, 2005.

Shadden, S. C., Lekien, F., Paduan, J. D., Chavez, F. P., and Marsden, J. E.: TheThe correlation between surface drifters and coherent structures based on high-frequency radar data in Monterey Bay, Deep Sea Res. II, 56, 161-172, 2009.

Sulman, M. H. M., Huntley, H. S., Lipphardt Jr., B. L., Jacobs, G., Hogan, P., and Kirwan Jr., A. D.: Hyperbolicity in temperature and flow fields during the formation of a Loop Current ring, Nonlin. Processes Geophys., 20, 883-892, doi:10.5194/npg-20-8832013, 2013.

Tallapragada, P. and Ross, S. D.: Particle segregation by Stokes number for small neutrally buoyant spheres in a fluid, Phys. Rev. E, 78, 036 308, doi:10.1103/PhysRevE.78.036308, 2008.

Titaud, O., Brankart, J. M., and Verron, J.: On the use of Finite-Time Lyapunov Exponents and Vectors for direct assimilation of tracer images into ocean models, Tellus A, 63A, 1038-1051, 2011.

Wiggins, S.: The Dynamical Systems Approach to Lagrangian Transport in Oceanic Flows, Annu. Rev. Fluid Mech., 37, 295$328,2005$. 